\title{
La Transmisión del Psicoanálisis en la Universidad: Una Experiencia $^{1}$ The Transmission of Psychoanalysis in an University Context: An Experience
}

\author{
Jaime Yasky Pérez²
}

\begin{abstract}
Resumen
En este artículo se presenta un particular modelo de formación universitaria en psiconálisis, exponiéndose algunas experiencias y problemas concretos que se han manifestado en la puesta en práctica de este modelo, tales como las dificultades encontradas por los alumnos en la operacionalización de la teoría de la técnica para poderse situar en el lugar de terapeutas y el efecto resistencial que puede tener la transferencia hacia el equipo formativo y hacia la teoría en el proceso de apropiación del conocimiento, resaltando la utilidad de este modelo y planteándose también algunas reflexiones que alcanzan el plano más general de la formación de psicólogos.
\end{abstract}

Palabras claves: psicoanálisis, enseñanza, escucha analítica, transferencia, resistencia.

\begin{abstract}
This paper presents a particular model of psychoanalytical training in a university context, exposing some experiences and problems that have come forth in the pursuit of that purpose, such as the difficulties experienced by students in trying to operationalize the theory of technic so they can situate themselves in the therapeutic position, and the resistencial effect that the transfer towards the training team and towards the theory can have to the process of taking possession of knowledge, stressing the usefullness of this model and proposing some reflections that can be useful to think the broader process of formation of psychologists.
\end{abstract}

Key words: psychoanalisys, teaching, analytic listening, transfer, resistance.

1 Escrito basado en parte de la ponencia “Lo psicoanalítico en la universidad y lo universitario en lo psicoanalítico" realizada en conjunto con Paulina Fernández y presentada en las II Jornadas Clínicas del CAPs de la Universidad de Chile, en Octubre del 2001.

2 Psicólogo, U. de Chile. Psicólogo Clínico Acreditado como Especialista en Psicoterapia. Miembro del Grupo de Estudios Psicoanalíticos de la Clínica de Atención Psicológica, CAPs. Docente Universidad Santo Tomás. Email: jaimeyasky@gmail.com 


\section{Introducción}

Este artículo tiene como objetivo compartir el trabajo de formación psicoanalítica que se realiza con alumnos de quinto año de la Carrera de Psicología de la Universidad de Chile a la luz de experiencias, problemas y reflexiones que han surgido en torno a éste.

Desde hace 8 años se realiza un trabajo en base a actividades clínicas y pedagógicas, acciones que conforman el dispositivo de formación que ofrece la Cátedra de Psicología Clínica ${ }^{3}$ a los alumnos que escogen cursar esta asignatura en su versión psicoanalítica. Estas actividades se realizan en la Clínica de Atención Psicológica (CAPs), que también depende del Departamento de Psicología, e incluyen además de las clases teóricas (en que se leen y discuten en conjunto escritos técnicos, psicopatológicos y casuística), la atención de pacientes por parte de los alumnos, la supervisión de tales procesos psicoterapéuticos y la participación de los alumnos en grupos operativos conformados a propósito de analizar el desarrollo de la tarea de atender pacientes.

La formación ofrecida asigna particular importancia a la atención de pacientes por parte de los alumnos, considerándose que es en la experiencia clínica, apoyada por las clases, la supervisión y los grupos operativos, que es posible que los alumnos puedan conocer con mayor intimidad la labor analítica.

¿Qué importancia tiene la revisión de esta experiencia formativa? Bueno, desde el punto de vista de la enseñanza del psicoanálisis, tiene importancia por que además de discutirse problemas similares a los enfrentados por otros esfuerzos de formación psicoanalítica, lo que en sí puede ser un aporte para aquellos que enfrentan dificultades parecidas, se plantea un modelo de formación analítica que se diferencia del clásico modelo de formación universitaria consistente en la enseñanza dogmática, escolástica e intelectualizadora, proponiéndose un modelo que intenciona una mayor asimilación y apropiación de lo aprendido por parte del aprendíz, lo que puede ser un aporte para aquellos que desde contextos universitarios están involucrados en similares esfuerzos de formación clínica.

Por otro lado, desde el punto de vista de la formación universitaria de psicólogos, se estima que esta experiencia y su discusión son importantes ya que tienen consecuencias que alcanzan al problema metodológico y ético más amplio de cómo enseñar psicología y como enseñar psicología clínica en particular: ¿que psicólogos produce y qué problemas trae consigo el modelo de formación imperante en nuestro medio, basada en el establecimiento de una relación unidireccional entre un profesor que imparte conocimiento y alumnos puestos en posición receptiva? pero también ¿qué es dable esperar como resultado y qué problemas traen consigo la implementación de un modelo formativo en psicología en que el trabajo con la experiencia subjetiva del aprendíz esté en un lugar central?

Como se pretende mostrar, el modelo de enseñanza del psicoanálisis que se expone pone énfasis en la coherencia entre lo que se enseña y la forma de enseñarlo, entre el contenido y la forma de transmitir dicho contenido, en la lógica de que el medio es el mensaje.

\section{El Objetivo de la formación}

El objetivo cardinal que se persigue con esta formación psicoanalítica es lograr que los alumnos hagan la experiencia práctica de

3 Cátedra de la Carrera de Psicología, de la Facultad de Ciencias Sociales de la Universidad de Chile, cuyo docente responsable es el Profesor Hugo Rojas O. 
realizar una "escucha analítica", o sea, que logren ejercitar la atención libremente flotante y desde tal postura mental puedan enfrentar la tarea de escuchar lo que sus pacientes les comunican. De esta manera se favorece la transmisión del psicoanálisis a través de ofrecer una exoestructura que propicia la ocurrencia y el reconocimiento de la actividad analítica en la propia experiencia del alumno, más que enfatizar una aproximación teórica o intelectualizadora.

¿A qué se refiere esto de hacer una escucha analítica? Al acto de interpretar lo que el paciente dice o comunica en base a prestar atención a determinados elementos de sus comunicaciones (expresiones y temáticas que escoje, entonación y puntuación de su discurso, su gestualidad, lapsus linguae, actos fallidos, sueños, etc.) que no parecen congruentes con sus intenciones y discurso consciente pero que permite ampliar y profundizar en la comprensión de las razones que determinan las problemáticas por las cuales consulta, al poner en palabra alguna verdad subjetiva resistida por su conciencia.

Importante para que esto ocurra es la capacidad del aprendíz de "extrañarse" o poner en suspenso una cadena de significantes (Rojas, 2001), o dicho en otras palabras, la capacidad de poner en suspenso todo tendencia a buscar algo en particular, todo criterio de selección del material ofrecido por el paciente, toda facultad crítica al escucharlo, permitiendo que la propia atención divage sin rumbo prestablecido y se fije espontáneamente en determinados elementos de las comunicaciones del paciente, posibilitando así que emergan otros cursos asociativos, otros significados, escuchando así otras cosas, que pudieran permitir conocer y entender mejor la realidad psíquica del paciente.

Se parte del supuesto que la realización de esta tarea no responde a la lógica de todo o nada, que en general, en una sesión "promedio", se ejercita esta función fragmentariamente, no de manera contínua.
Visto así, es posible que un terapeuta novel logre hacer la experiencia de escuchar de esta forma, tal vez con dificultades para percatarse de que lo hizo y sin garantía de que pueda repetirlo,o que pueda sostenerla con la frecuencia suficiente para aportar lo necesario para mantener en curso un proceso analítico. Se propicia primero la ocurrencia de esta experiencia, luego su reconocimiento, y posteriormente su repetición y su sostenimiento lo más posible dentro de las condiciones clínicas y pedagógicas en que se trabaja.

\section{El dispositivo formativo}

Pichón-Rivière (1972) entiende al aprendizaje como instrumentalización de la teoría, como posibilidad de operar sobre la realidad conforme a un determinado saber acerca de ella. Visto así, un punto clave para que los alumnos puedan atender a sus pacientes es que logren apropiarse instrumentalmente de la teoría, lo que se logra al sostener una postura crítica y creativa frente a ésta. Como se verá más adelante, justamente estas facultades críticas y creativas de los alumnos se ven dificultadas por la transferencia no elaborada respecto a la institución del profesor y otras figuras que representan un ideal, un deber ser inalcanzable.

Pero Pichón-Rivière (1972) también concibe el aprendizaje como praxis, es decir, plantea que sólo se produce en la medida que se opera con la realidad. Desde este ángulo, el asunto central pasa a ser cómo se diseña y propicia una experiencia práctica, en este caso la atención clínica de pacientes, que sea contenida por un dispositivo que posibilite el despliegue de una postura crítica y creativa respecto a los lineamientos teóricos que se entregan para que resulten útiles para afrontar la praxis clínica que se realiza.

¿El dispositivo ofrecido apunta efectivamente en esa dirección? Revisando las actividades que se hacen se puede decir que en 
las clases se fomenta la participación y discusión activa de los alumnos en la asimilación de los conceptos teóricos estimulando a que ellos mismos lean, presenten las lecturas a tratar y problematicen lo leido, con la participación del docente como apoyo, aportanto aclaraciones y profundizando en los contenidos.

Tanto la supervisión como los grupos operativos colaboran en establecer el nexo entre teoría y praxis y en desarticular los problemas que experimentan los alumnos para hacer tal integración. El en espacio de supervisión los alumnos revisan junto a su supervisor lo ocurrido en el tratamiento de sus pacientes, analizándose sus contenidos, dándose indicaciones de cómo proceder, discutiéndose dudas tanto técnicas como de diagnóstico y señalándose aquellas tendencias del terapeuta que aportan u obstaculizan la labor analítica. Esta tiene efectos de apoyo pues es un lugar en que el alumno participa de una relación con alguien que literalmente lo contiene: lo ayuda a contener las ansiedades y emociones despertadas a propósito del caso, permitiéndole pensar el caso y pensarse en el caso, recibiendo además "cosas" -elementos técnicos- que le permiten lidiar de mejor forma con las dificultades encontradas en la atención de su paciente. El supervisor, quiéralo o no, por la natural tendencia a la transferencia que el encuadre propicia, ofrece un modelo a seguir respecto a cómo operar como analista, ya que al supervisar opera analíticamente, por lo que también la supervisión tiene efectos de apoyo. La identificación con el modelo permite a los alumnos manejar mejor la angustia, permitiendo mayor margen para pensar, aunque en este espacio no se resuelvan los determinantes intrapsíquicos de ésta, y como se verá más adelante, también incluye el riesgo de propiciar una relación regresiva que inhiba una apropiación crítica y creativa de la técnica.

Pero la supervisión también tiene una dimensión analítica: el supervisor formula interrogantes y hace señalamiento que apuntan a precisar cómo es que el caso pudiera estar estructurado a imagen y semejanza de las ansiedades, deseos, defensas y en general de la contratransferencia del aprendíz, abriendo así la posibilidad de que éste piense cómo está involucrado en el caso. Si se constituyen resistencias al trabajo de supervisión, esta puesta en cuestión también pueden incluir la forma en que el aprendíz participa de la instancia de supervisión, lo que puede iniciar la desarticulación de esquemas de relación regresivas a través de tomar conciencia de éstos, en la medida que tales esquemas obstaculicen el desempeño de la función analítica por parte del aprendíz. Esta instancia no tiene como propósito profundizar en el origen o razones detrás del aporta personal que el terapeuta hace al caso que atiende, estos señalamientos e interrogantes sirven para tomar conciencia que indefectiblemente se está involucrado en concepción del caso, en la determinación de lo que está experimentando con el paciente, lo que es un enorme aporte para la compresión de la tarea analítica y sus dificultades.

Por otro lado, los grupos operativos están pensados como instancia para dar cabida a las ansiedades y dificultades que experimentan los alumnos en torno a la tarea de ubicarse como terapeutas, a través de intentar su resolución analítica. Se analizan las ansiedades inconscientes y las defensas del grupo ante éstas, por lo que tiene un efecto económico importante en el sentido de ampliar las posibilidades de pensar menos impulsivamente. En este dispositivo se analiza lo que surge en el grupo tomando como punto de referencia la tarea clínica, ya que su objetivo es justamente apoyar la tarea clínica a través de analizar y desarticular las dificultades y resistencias que se presentan a propósito de su realización. Su objetivo no es tratar psicoterapéuticamente al grupo o sus integrantes, lo que no impide que el trabajo que se hace pueda tener efectos terapéuticos. 
Por tanto, ni la supervisión ni el grupo operativo llegan al lugar que le corresponde al propio análisis; se señalan dinámicas defensivas de los alumnos, pero exclusivamente en función de desarticular resistencias u obstáculos para la realización de la labor clínica. Desde el encuadre en que se opera se propicia, recomienda y hasta se sirve de puente para aquellos alumnos que quieren iniciar su propio análisis, pero no se les pide ni exige hacerlo, pues además de no tener las atribuciones para hacerlo, no se considera ni lícito ni conducente regular el análisis del propio aprendiz

El propio análisis del aprendíz es fundamental por varias razones, tanto personales como relativas a la formación psicoanalítica, pero entre ellas hay una que nos concierne particularmente. Una consecuencia pedagógica esperable - aunque no garantizable - del propio análisis es el discernimiento de que lo aprendido teórica y técnicamente es necesariamente una interpretación y que, dejando de lado las asimetrías en cuanto a experiencia y en cuanto a grado de conocimiento, ese otro al que se le supone cierto saber especial, básicamente no puede sino ser un par en cuanto a tener el mismo tipo de relación con el saber - incompleta, parcial- que la que él mismo puede aspirar a tener.

\section{Algunas experiencias y problemas}

Presentados los propósitos y la metodología de este dispositivo, a continuación se revisan algunas experiencias que permiten analizar cómo resulta en los hechos su puesta en práctica.

Juntando impresiones acerca del discurso de los alumnos que han pasado por la cátedra en estos años, se ha observado que desde los alumnos surjen dificultades relacionadas con las reglas que deben seguir o resguardar para quedar "correctamente sentados" en el lugar del terapeuta: nos referimos a la regla de neutralidad, a la regla de abstinencia, a la regla fundamental y a otros elementos técnicos que encuadran la labor analítica. Por ejemplo, surgen inquietudes tales como "¿debo hablar o guardar silencio en sesiones?” “¿debo responder las preguntas del paciente?" "¿debo decirle al paciente qué tiene?" “¿cómo saludo al paciente?" “¿al paciente lo trato de Ud. o lo tuteo?" “ ¿estamos haciendo psicoterapia o psicoanálisis?" "¿cómo hago para que el paciente asocie libremente?” “ ¿cómo se hace la atención libremente flotante?"

Estas interrogantes, planteadas con mayor o menor grado de claridad, van de la mano con sentimientos de inseguridad y confusión respecto a cómo cumplir o hacer cumplir estas importantes reglas, lo que se acompaña de una búsqueda frenética de referentes concretos que ayuden a poner en práctica lo que la teoría de la técnica manda. Es así que, por lo menos para una parte importante de los alumnos, la enseñanza teórica de la técnica, que supuestamente debiera orientarlos y posibilitar que puedan operar analíticamente, se constituye en una serie de mandatos incumplibles, despertando ansiedades persecutorias relativas a su desempeño como terapeutas: temen ser descalificados ya sea por el profesor, por sus supervisores, por los coordinadores de los grupos operativos, por sus compañeros o por sus mismos pacientes.

Para muchos alumnos la transferencia con la teoría misma, en tanto conceptos no operacionalizados, no asimilados, y la transferencia con los portadores de ésta, en tanto fenómenos no analizados, interfieren más que facilitan las posibilidades que tienen de hacer una experiencia analítica. Se observa entonces que la teoría, aquello que debería servir para orientar la práctica se convierte en regla de oro, en un deber ser incumplible dada la acentuación de la severidad superyoica, constituyéndose una resistencia para la puesta en práctica del método psicoanalítico. 
Frente a estas ansiedades, los alumnos tanto individual como grupalmente se defienden con distintos mecanismos, que siguiendo los planteamientos de Bleger (1961), en tanto se vuelven estereotipados, paralizan el proceso de enseñanza - aprendizaje. Algunas estrategias defensivas observadas que alcanzan un nivel importante de frecuencia y estereotipia son la mitificación e imitación de la figura del profesor, el refugio en el dominio intelectual de los conceptos, la descalificación propia o de los pacientes y la huida de la tarea.

Existen otras variables que inciden sobre el grado de dificultades que experimentan los alumnos. Están enfrentando una tarea nueva, la de atención clínica de un paciente, para la cual casi no cuentan con experiencia práctica previa ni algún tipo de estrategia previa de aproximación con niveles de dificultad progresivos. Están cerca de egresar y por tanto para muchos la atención de pacientes representa una prueba de si tienen o no lo requerido para enfrentar el mundo laboral. Si además a ésto sumamos lo incitante que resulta el encuadre pedagógico clásico, con la institución del profesor en posición del "sujeto supuesto saber" (Lacan, 1967) para la regresión y transferencia, no resulta extraño que surjan estas dificultades en la tarea de apropiación y puesta en práctica de los conceptos técnicos, o sea, en la tramitación simbólica de la experiencia clínica.

Este contexto intrapsíquico del aprendíz - que puede ser predecible pero no por ello menos preocupante - caracterizado por la sensación de falta y por el opacamiento o flaqueo de sus recursos, lo lleva a recurrir a la literalización, a apegarse a la forma, a imitar y encarnar de manera concreta la imagen del que supuestamente sabe unívoca e inequívocamente de qué se trata hacer análisis (Freud, otros autores, o sus delegados "terrenales", el profesor y los supervisores) como forma de solución.
Otro elemento que emerge en el discurso de los alumnos es la necesidad de terapia. Con distintos matices de reflexión previa, esta necesidad es formulada, expresada e inclusive concretada por algunos alumnos justamente en este momento, lo que sugiere una conexión latente con las dificultades enfrentadas con la tarea clínica, aunque también hay otros factores que precipitan esta decisión, tales como el momento vital en el que están, en el umbral de egreso hacia el mundo profesional, la influencia del encuadre formativo de la cátedra sobre la necesidad de un análisis propio y el paulatino desarrollo de una cultura de validación de la psicoterapia como instancia ayuda y desarrollo personal en el estudiantado o la comunidad de la escuela de psicología.

El deseo de terapia no está exento de expectativas mágicas. Algunos alumnos ven en ésta una solución instantánea a los males que experimentan en su proceso de aprender y practicar el psicoanálisis, como si el mero hecho de asistir y hablar de sus problemas en terapia bastara para que éstos se resolviesen. Analizar con los alumnos - en el contexto de los grupos operativos - su motivación hacia su propia terapia, tiene efectos terapéuticos y pedagógicos, en la medida que ayuda a reconocer y descolocar estereotipos de subordinación, dependencia o impotencia en que se sitúan los alumnos al abordar la tarea de aprender a ser terapeutas, y que se articulan con el deseo de terapia.

El panorama recién presentado podría llevar a alarma y a precipitar la conclusión de que las dificultades que experimentan los alumnos son resultados directo de ser sometidos a un nivel de exigencia excesivo. Pero llegar a esta conclusión desconsidera el hecho de que lo observado, las dificultades de aprendizaje y ansiedades de los alumnos se hacen patentes justamente en virtud de la existencia de un dispositivo que posibilitan que emerjan, que se manifiesten. Instancias tales como la supervisión y el grupo operati- 
vo están pensadas justamente para ofrecer oido, contención y análisis a lo que surge de los alumnos mismos al verse enfrentados a las exigencias de realizar la tarea clínica, haciéndose así observables, concientes, dificultades que en menor o mayor medida probablemente también están presente en otras cátedras e instancias formativas de la carrera, instancias que por no contar con canales idóneos para dar curso a cómo se involucran subjetivamente los alumnos en la tarea de apropiación de lo aprendido, probablemente no constatan esta realidad ${ }^{4}$.

\section{Revisión de los resultados, conclusiones y alcances}

La experiencia con varias generaciones de alumnos indica que sí es posible lograr los objetivos formativos que nos planteamos, lo que se evidencia tanto en lo que reportan los alumnos como en el hecho de que a partir de esta experiencia formativa muchos aclaran o afianzan su interés por seguir formándose en psiconanálisis. Además, cursar la cátedra ha resultado una experiencia novedosa y enriquecedora para muchos, siendo para la mayoría la primera vez que participan de un proceso formativo que gira en torno a elaborar su experiencia, en que han podido y tenido que exponer lo que piensan y hacen, asumiendo mayor autonomía y responsabilidad por ello.

Sin embargo, también es cierto que los resultados no son homogéneos, que entre aprendíz y aprendíz, así como entre los diferentes grupos que año a año ingresan a este dispositivo se notan diferencias en los resultados.

Una característica observada en los alumnos que logran hacer una experiencia analí- tica es la capacidad ${ }^{5}$ de tomar la experiencia propia y ajena como materia pensable. El estar o haber estado en un proceso analítico como paciente no ha demostrado ser una condición necesaria para presentar esta capacidad, pues no todos los que están o han estado en análisis la presenta, ni todos los que la presentan están o han estado en análisis.

Además de la incidencia de las diferencias individuales, los grupos-cursos vienen constituidos de diversas formas, vienen ya con historia, con dinámicas peculiares tales como rivalidades y competencias internas, polarización y personificación de posturas, asignación de roles, etc. que implican enfrentar desafíos diferentes cada año. Se notan tendencias grupales que aportan a la realización de la tarea, tales como el interés y dedicación serios y la competencia constructiva y compenetrada en la tarea, como también tendencias que obstaculizan la realización de la tarea, tales como la polarización de rivalidades entre subgrupos, la evasión conjunta de la tarea, el apoyo a liderazgos con posturas oposicionistas o contestatarias, etc.

Es cierto que los espacios antes reseñados (la clase, la supervisión, el grupo operativo) contienen y posibilitan que los aprendices piensen su quehacer clínico, elaboren su experiencia y aprendan de ésta, pero también es cierto que la efectividad de estos recursos está siempre en función de la dimensión de la tarea. Desde el punto de vista económico el problema es que, dadas las diferencias en cuanto a equilibrio psicológico, lo que es suficiente para un alumno en términos de contención no necesariamente lo es para otro. Lo mismo ocurre si lo vemos desde el punto de vista dinámico: las dinámicas intersubjetivas profesor-alumno, supervisor-supervisando,

4 sería interesante indagar cómo se manifiestan estas dificultades en otras intancias formativas de la carrera, y qué relación tienen éstas con prácticas y fenómenos comunes en el alumnado tales como el ausentismo, la copia, el fracaso académico y el aprendizaje memorístico.

5 este tema ha sido investigado y planteado de alguna forma por Winnicott (1967) y por Heimann (1977): el concebir la actividad analítica como especialización de ciertas funciones mentales normales. 
coordinadores-grupo son particulares, por lo que la mayor afinidad que se establezca entre un profesor, supervisor o coordinador de grupo operativo con un determinado aprendíz, y la consecuente utilidad que tenga tal relación para éste, no es necesariamente extrapolable a otros.

El nivel de dificultades experimentado por un aprendíz en el proceso formativo depende en grado importante de sus condiciones psicológicas (su neurosis o psicopatología), pero esto no ocurre en abstracto: el precisar la existencia de resistencias al aprendizaje de parte de los alumnos no exime al encuadre formativo de tener que dar cuenta de cómo propicia, contiene y da curso a las esperables dificultades que ocurren en éste. Esto último plantea un desafío para todos los que cumplen roles en este dispositivo formativo, ya que las dificultades experimentadas por los alumnos en la consecusión de la tarea formativa también reflejan las limitaciones, tanto individuales (de los profesores, supervisores o coordinadores grupales) como del encuadre formativo para propiciar la consecusión de los objetivos formativos.

El hecho de que los buenos resultados no abarcan todos los alumnos junto a la repetición de las dificultades antes planteadas han llevado a preocuparse más por velar por que se den las mejores condiciones posible para enfrentar la tarea clínica. Estas condiciones incluyen la selección de los pacientes que se asignan a los alumnos en función de la gravedad de la problemática que presentan, y el prestar atención a las condiciones psicológicas de los alumnos que enfrentar la tarea clínica, preocupándose de no exponer a los rigores de la actividad clínica a alumnos que ya estén exigidos en su capacidad de pensar por sus propias problemáticas y que por tanto no dispongan de un margen de capacidad de trabajo psicológico suficiente para la atención de un paciente.
Por último, también importa el nivel de entrenamiento y experiencia de quienes conforman el equipo formativo (docente, supervisores y coordinadores de grupo operativo) cuya mayor o menor capacidad de trabajo también determina que se logre enfrentar exitosamente la tarea formativa. $\mathrm{Al}$ respecto, además de existir un espacio semanal en que los miembros del equipo formativo pueden tratar grupalmente las dificultades que enfrentan al realizar sus tareas formativas, finalizado el periodo académico se desarrolla habitualmente una revisión crítica del funcionamiento de las diferentes actividades de la cátedra, en que los encargados de cada actividad, las clases, las supervisiones y los grupos operativos, presentan los resultados y dificultades experimentadas con el objetivo de analizarlos conjuntamente, aprender de la experiencia y así proyectar de mejor forma la labor que se realizará el siguiente periodo. Fruto de este trabajo de revisión anual es que se han pensado e implementado muchos de los cambios que han redundado en la conformación actual del dispositivo formativo, tales como la inclusión hace algunos años de los grupos operativos, la supresión de ciertas categorías de evaluación del aprendíz relativas a su rendimiento o talento como clínico, la supresión de la atención en espejo de pacientes, la disminución de la cantidad de aprendices por supervisor y la supresión de la supervisión de pacientes con el profesor en el horario de clases.

Respecto a los alcances que se pueden desprender de lo anteriormente expuesto, primero es importante destacar que es posible poner en práctica y obtener resultados positivos con un modelo de formación que intenciona una mayor asimilación y apropiación de lo aprendido por parte del aprendíz a través de ofrecer instancias de elaboración de la forma en que está involucrado en la tarea de aprender. Esto propicia el despliegue de facultades críticas y creativas que redundan en el desarrollo de una postura de mayor autonomía, en que el aprendíz se apropia de lo aprendi- 
do, ajustando lo que aprende a sí mismo, a su experiencia subjetiva.

La experiencia de este autor, tanto como alumno, como docente universitario en psicología por más de doce años y como partícipe de dos grupos de trabajo centrados en la revisión y postulación de modelos de formación en psicología en nuestro medio (ver Yasky, 1999), indica que no hay mucha variabilidad en cuanto a la metodología de enseñanza de la psicología, preponderando un modelo de formación universitaria clásico que asume tácitamente que la enseñanza consistente en el aprendizaje intelectual del cuerpo teórico de la disciplina, que la figura del profesor es la central en el proceso de enseñanza, que los resultados del proceso dependen fundamentalmente de la mejor o menor capacidad que tenga éste de administrar y distribuir su conocimiento, y que da por descontado que frente al profesor está un alumno estándar, en posición receptiva, a quien se le exigen credenciales académicas y tal vez certificación de que carece de contraindicaciones psicopatológicas importantes, lo que sería suficiente para esperar que ocurra una exitosa experiencia formativa.

Si bien es cierto que en atención a los cambios cuantitativos y cualitativos experimentados por la población universitaria de nuestro medio en los últimos dos decenios se ha incrementado la preocupación por conocer las características de los alumnos actuales y así ajustar la metodología de enseñanza a tales cambios (ver por ejemplo Miranda, 2000), las modificaciones realizadas en los programas de formación en psicología no han afectado mayormente las actividades que constituyen el núcleo de la formación en psicología ${ }^{6}$.

Un problema metodológico y ético que afecta particularmente la enseñanza de la psicología, dado que su objeto de estudio es equivalente a la subjetividad del aprendiz, es el divorcio entre lo que el alumno aprende a través de escuchar a un profesor o leer un libro y lo que experimenta en sí mismo. Por ejemplo, es común la experiencia de asistir a una clase de psicopatología en que se aborda el tema de la angustia, y al escuchar la descripción de la experiencia de angustia el alumno se siente identificado con lo que se describe, movilizándose en él inquietudes que no son elaboradas, no pudiendo articularse lo aprendido en clase con la propia experiencia (salvo conversaciones de pasillo con algún profesor de confianza o hablando con sus pares), tendiendo entonces a incorporarse un conocimiento que resulta ajeno, $\mathrm{di}$ vorciado de la propia experiencia, sin posibilidad de abordar de manera constructiva la involucración subjetiva del aprendíz con lo que aprende, no pudiendo tratarse inquietudes tales como "¿qué es la angustia en mi experiencia?" “ ¿significa que tengo algo malo o estoy mal?" “¿se habrán equivocado al dejarme entrar a la carrera?" "¿será mejor suprimirlo o negarlo para evitar problemas?"

¿Es el tipo de psicólogos que resulta de este modelo de formación equivalente al que se pretende lograr según lo expresado, por ejemplo, en el perfil de egreso? Esta materia requiere de un mayor análisis, pero se estima que la experiencia formativa reseñada tie-

6 los cambios realizados, si bien apuntan a proveer instancias de mayor participación de los alumnos en el proceso de aprendizaje, reflejando la influencia de la concepción constructivista del proceso de aprendizaje, siguen siendo cambios marginales, consistentes en talleres y actividades colaterales que tienen como objetivo mitigar deficiencias o problemas detectado en el perfil de ingreso, tales como deficiencias en el nivel de lecto-escritura, en habilidades cognitivas, problemas actitudinales, vocacionales, de madurez emocional y/o moral. 
ne alcances en la problematización en este tema.

Según Freud (1937, p. 249) “... analizar sería la tercera de aquellas profesiones imposibles' en que se puede dar anticipadamente por cierto la insuficiencia del resultado. Las otras dos, ya de antiguo consabidas, son el educar y el gobernar". De la experiencia de formación en psicoanálisis expuesta se desprende que la imposibilidad de garantizar la suficiencia del resultado es consecuencia de asumir una opción metodológica y ética de cómo enseñar psicoanálisis, opción que entre otras cosas pasa por reconocer que los resultados de esta actividad formativa depende principalmente de lo que ocurre en el aprendíz, renunciando como formador a la ilusoria tentación de tener bajo control sus resultados y disponiéndose a colaborar en lo necesario para que ocurra.

\section{Referencias Bibliográficas}

1. Freud, S. (1937): Análisis terminable e interminable. En Obras Completas, Vol. XXIII. Amorrortu Editores, Buenos Aires.

2. Heimann, P. (1977): Further observations on the analyst's cognitive process. En "About Children and Children-NoLonger". Ed. Routledge, London.

3. LACAN, J. (1967-68): Seminario 15 "El acto psicoanalítico". En Bibliotecas de Psicoanálisis, http://www.psicoanalisis.org/. Consultado el 30 de Julio del 2005.

4. Miranda, G. y otros (2000): Psicología contemporánea; disciplina, profesión, formación. Ed. Universidad Católica Cardenal Raúl Silva Henriquez, Santiago.

5. Pichon - Rivière, E., EN COlAbORACión CON
Ana P. De Quiroga (1972): Grupo Operativo e intervención institucional. En Obras Completas. Ed. Nueva Visión, Buenos Aires.

6. Rojas, Hugo (2001): Comunicación Personal. Departamento de Psicología, Universidad de Chile. Santiago, Chile.

7. WinnicotT, D.W. (1967): “Mirror-role of Mother and Family in Child Development" en "Playing and Reality". Ed. Tavistock/Routledge, London.

8. YASKY, J. (Ed.) (1999): Masificacion de la educacion universitaria en Chile: desafios para la Psicologia. Documentos de estudio $n^{\circ}$ 51. Ed. Universidad Católica Cardenal Raúl Silva Henriquez, Santiago.

Fecha Recepción Artículo: 21 de septiembre 2005

Fecha Evaluación Final: 23 de junio 2006 